\title{
O VIVIDO DOS ENFERMEIROS NO CUIDADO AO PACIENTE ONCOLÓGICO*
}

Anna Maria de Oliveira Salimena1, Simone de Rezende Teixeira ${ }^{2}$, Thaís Vasconselos Amorim ${ }^{3}$, Andyara do Carmo Pinto Coelho Paiva ${ }^{3}$, Maria Carmen Simões Cardoso de Melo ${ }^{4}$

RESUMO: Esta pesquisa teve como objetivo conhecer as percepções e sentimentos de enfermeiros de um hospital oncológico de referência em diagnóstico e tratamento de pacientes com câncer na Zona da Mata Mineira. Foram nove participantes pesquisados nos meses de janeiro e fevereiro de 2012 mediante entrevista aberta. Da análise compreensiva emergiram as unidades de significado: O movimento de colocar-se no lugar do outro e A contradição dos sentimentos na prática do cuidado. Consideramos que o cuidado do enfermeiro pode ser compreendido em suas facetas técnicas, ético e humano, uma vez que possibilita o aprofundamento das relações interpessoais entre aqueles que cuidam e os que são cuidados.

DESCRITORES: Cuidados de enfermagem; Neoplasias; Serviço hospitalar de oncologia.

\section{NURSES' EXPERIENCE IN THE CARE OF THE ONCOLOGY PATIENT}

ABSTRACT: This research aimed to investigate the perceptions and feelings of nurses in a hospital which is a center of excellence in the diagnosis and treatment of patients with cancer in the Zona da Mata Mineira region. Through open interviews, nine people participated in the research, in the months of January and February 2012. The following units of meaning emerged from the comprehensive analysis: The movement of putting oneself in another's position and The contradiction of feelings in care practice. The authors consider that the nurse's care may be understood in terms of its technical, ethical and human aspects, as these make it possible to deepen interpersonal relationships between those who care, and those who are cared for. DESCRIPTORS: Nursing care; Neoplasms; Hospital oncology service.

\section{LO QUE VIVEN LOS ENFERMEROS EN EL CUIDADO AL PACIENTE ONCOLÓGICO}

RESUMEN: Esta investigación tuvo el objetivo de conocer las percepciones y sentimientos de enfermeros de un hospital oncológico de referencia en diagnóstico y tratamiento de pacientes con cáncer en la Zona da Mata Mineira. Fueron nueve participantes acompañados en los meses de enero y febrero de 2012 por medio de entrevista abierta. Del análisis comprensivo resultaron las unidades de significado: El movimiento de colocarse en el lugar del otro y La contradicción de los sentimientos en la práctica del cuidado. Se considera que el cuidado del enfermero puede ser comprendido en sus aspectos técnico, ético y humano, una vez que posibilita la profundización de las relaciones interpersonales entre los que cuidan y los que son cuidados. DESCRIPTORES: Cuidados de enfermería; Neoplasias; Servicio hospitalar de oncología.

\footnotetext{
*Recorte do trabalho de conclusão de Curso da Faculdade de Enfermagem da Universidade Federal de Juiz de Fora - FACENF UFJF, 2012.

${ }^{1}$ Enfermeira. Doutora em Enfermagem. Professora da FACENF UFJF. Coordenadora do Programa de Pós-Graduação em Enfermagem da UFJF.

${ }^{2}$ Enfermeira.

${ }^{3}$ Enfermeira. Mestranda pelo Programa de Pós-Graduação em Enfermagem da UFJF. Bolsista CAPES.

${ }^{4}$ Enfermeira. Doutora em Enfermagem. Professora da FACENF UFJF.
}

Autor correspondente:

Anna Maria de Oliveira Salimena

Universidade Federal de Juiz de Fora

R. M.al Cordeiro de Faria, 172 - 36081-330 - Juiz de Fora-MG-Brasil

E-mail: annasalimena@terra.com.br
Recebido: 28/08/2012

Aprovado: 21/02/2013

Cogitare Enferm. 2013 Jan/Mar; 18(1):142-7 


\section{INTRODUÇÃO}

No Brasil, as neoplasias ocupam o segundo lugar nas causas de morte por doença, segundo o Sistema de Informação sobre Mortalidade (SIM), sendo reconhecidas como um problema de saúde pública ${ }^{(1)}$. Consoante com a Organização Mundial de Saúde (OMS), estima-se que no ano de 2030 sejam registrados 27 milhões de novos casos de câncer mundialmente, 75 milhões de pessoas vivendo com a doença anualmente e 17 milhões de mortes $^{(2)}$, o que reflete a necessidade de profissionais qualificados para dar subsídios no tratamento, reabilitação, cura e cuidados paliativos.

A essência do trabalho dos enfermeiros consiste na prestação de cuidados à saúde, envolvendo aspectos de ordem bio-psico-socio-espiritual. Portanto, no seu cotidiano de trabalho vê o indivíduo no processo de adoecer, por meio de perspectiva holística em multidimensionalidade. A partir disso, têm-se diversas concepções acerca do servir ao outro. Essa atitude pode ser compreendida como "uma ação e comportamento de assistir, administrar e ensinar com zelo, mantendo o bem estar"(3:22).

A equipe de enfermagem, que atendem aos pacientes fora de possibilidade de cura, precisa ter, além de habilidade técnica para prestar cuidados físicos, sensibilidade nos aspectos psíquicos envolvidos, pautado na ética e na humanização ${ }^{(4)}$. Muitas vezes as neoplasias estão associadas a sofrimento e dor, o que trás ao paciente grande instabilidade emocional durante a vivência da doença e de seu tratamento. Acrescentam-se seus medos, angústias e incertezas referentes à patologia e às alterações que poderão ocorrer durante o processo terapêutico.

Sendo assim, a vivência de situações difíceis em uma unidade de oncologia pode despertar uma infinidade de sentimentos ao cuidador, com repercussões de ordem física e emocional ${ }^{(5)}$. Entretanto, a maneira de lidar com as diferentes circunstâncias dependerá da percepção individual.

Dentre as situações promotoras de medos e incertezas aos profissionais, destacam-se as que se associam à natureza da doença, à complexidade do cuidado e o grande envolvimento com os pacientes e seus entes queridos. Deste modo, os enfermeiros buscam maneiras de se comportar frente ao sofrimento e perspectiva da morte no cotidiano laboral ${ }^{(6)}$. Assim, é imprescindível o preparo emocional do profissional a fim de oferecer a melhor assistência possível, considerando o valor terapêutico de um ambiente tranquilo e seguro para a equipe, pacientes e familiares.
Diante do exposto, julga-se importante a reflexão sobre os aspectos transpessoais envolvidos no diagnóstico e tratamento do câncer, na perspectiva dos profissionais que assistem diretamente os pacientes acometidos. Para isso, é necessário conhecer as percepções e sentimentos dos enfermeiros que atuam em um hospital oncológico, o que se constitui o objetivo deste estudo.

\section{MÉTODO}

Trata-se de uma pesquisa de abordagem qualitativa, tendo sua análise apoiada no referencial teórico-metodológico de Martin e Bicudo ${ }^{(7)}$. Considera-se que os fenômenos do vivido dos enfermeiros de um hospital oncológico desvelam-se a partir das descrições das experiências dos depoentes.

O projeto foi encaminhado ao Comitê de Ética em Pesquisa da Universidade Federal de Juiz de Fora, para análise e deferimento quanto ao cumprimento dos aspectos éticos e legais, conforme Resolução n. 196/96 ${ }^{(8)}$ e foi aprovado segundo parecer n. 245/2011. A pesquisa de campo foi realizada nos meses de janeiro e fevereiro de 2012 em um hospital de referência no combate ao câncer na região da Zona da Mata Mineira, que ocupa lugar de destaque na assistência hospitalar aos pacientes oncológicos.

Os participantes foram 8 enfermeiras e um enfermeiro, tendo como critério de inclusão aqueles que tivessem pelo menos um ano de atuação na instituição do estudo. Para a coleta dos dados valeu-se da entrevista aberta baseada no referencial de Carvalho, por ser uma forma de o participante adentrar na verdade de si mesmo, sem qualquer não verdade ou preconceito ${ }^{(9)}$. $\mathrm{O}$ encontro foi agendado previamente, de acordo com a disponibilidade dos enfermeiros, e as informações foram gravadas, a partir da seguinte questão norteadora: Fale sobre os sentimentos que você vivencia assistindo/trabalhando com pacientes oncológicos. Com a finalidade de resguardar o anonimato dos depoentes, as entrevistas foram denominadas de "E", seguidas do número correspondente à sequência das falas.

Após a transcrição dos depoimentos realizou-se leitura exaustivamente com o objetivo de identificar o conteúdo exposto pelos enfermeiros seguindo os passos do referencial elegido: as descrições capazes de refletir o sentido ampliado; a convergência destas em unidades de significados; a interpretação das falas em linguagem psicológica e a consolidação das unidades em direção à análise compreensiva ${ }^{(7)}$. 


\section{RESULTADOS}

A partir do relato dos entrevistados foi possível apreender que é difícil não se deixar abalar psicológica e emocionalmente frente às exigências que perpassam os cuidados técnico-assistenciais, sendo evidente a comoção vivenciada por esses trabalhadores em sua rotina. Também se evidenciou que embora cuidar de alguém com uma patologia grave apresente-se como tarefa difícil, a assistência ao paciente com câncer é permeada por sentimentos positivos, como a gratificação, a qual o pouco que a equipe pode oferecer em alguns momentos é visto como muito pelos pacientes e familiares. Nesse sentido, os profissionais sentem o reconhecimento pelo trabalho realizado.

Foram extraídas, a partir dos depoimentos, as estruturas essenciais que permitiram emergir as unidades de significados, a seguir apresentadas com o recorte de alguns depoimentos

\section{O movimento de colocar-se no lugar do outro}

Para o enfermeiro a assistência prestada ao paciente não se constitui apenas em executar tarefas técnicas, mas em ter habilidade de saber escutar, falar e perceber que uma simples conversa com o paciente faz com que a assistência se dê com qualidade, conforme este recorte:

[...] você pode resolver o problema do paciente simplesmente com um diálogo, não é só fazer, administrar uma medicação, fazer um curativo. (E7)

Verifica-se também que quanto mais jovem é o paciente, mais difícil é para o enfermeiro lidar com as situações de dor e sofrimento, como apresentado neste depoimento:

[...] você tentar se aproximar mais daquela criança é dificil, porque envolve muitas coisas [...] é no dia-a-dia, é o contato, é a visita diária, é uma conversa [...]. (E7)

$\mathrm{O}$ ato de se colocar no lugar do outro não é uma tarefa fácil, como exposto:

[...] ser empático e estar se colocando no lugar daquela pessoa. (E2)

[...] assim acaba que a gente se coloca sempre no lugar deles, vê eles assim sofrendo. (E3)

No começo eu acho que fica mais difícil mesmo [...] principalmente quando lida com criança [...] isso trás um transtorno maior para gente. (E5)

É difícil, mas tentam se colocar no lugar do paciente [...] aquela sensação de estar sozinho, de não ter ninguém, seja uma família, então você procura ficar mais perto. (E7)

\section{A contradição dos sentimentos na prática do cuidado}

Os enfermeiros estão envolvidos por sentimentos, que variam desde o sofrimento ao lidar com paciente até a satisfação profissional, como expresso pelos depoentes:

[o cuidado] tem o seu lado positivo, que é a receptividade em relação ao paciente. A família, que muito pouco a gente faz, para eles é muito [...]. É um local que a gente sente que realmente faz a diferença, porque eles realmente precisam muito do nosso cuidado. (E1)

Os sentimentos são todos misturados, tem felicidade, tem alegria, tristeza, angústia, decepção, são todos os sentimentos que a gente convive o tempo todo. (E9)

O sentimento de gratificação, tendo em vista as etapas de recuperação do paciente e a cura da neoplasia, também é destacado pelos participantes:

[...] quando ele levanta da cama, consegue ficar fora do oxigênio, passa a se alimentar, por exemplo, sem uma sonda [...] cada etapa dessas para a gente é uma vitória! (E1)

Você tem um extremo, às vezes interna paciente que vem, faz uma radioterapia, então ele está bem [...] tem um efeito colateral ou outro que supera assim com tranquilidade. É muito fácil, quando eles vão embora uma felicidade que dá. (E3)

O sentimento é de prazer. Eu faço como um hobby [...] aquele paciente que você vê ele lutando, ele quer vencer! E você vê ele conseguindo os objetivos, isso é bom, é gostoso! (E4)

[...] tem sentimento de felicidade quando a pessoa vai embora, vai para casa [...]. ( E6)

Para os enfermeiros, que são expostos diariamente a situações de dor, sofrimento e morte, torna-se difícil não se abalar psicológica e emocionalmente diante destas situações, como expresso: 
Você vê essa pessoa que melhorou, que lutou, que venceu! Aí, vê ele voltando, já com uma recidiva da doença [...]. Aí eu sofro! (E4)

Às vezes, realmente a gente sofre mesmo. Além de sermos profissionais, nós somos seres humanos, não vejo vergonha nenhuma, o paciente ir a óbito, você chorar. O tratamento é longo, você está ali acompanhando desde o início. (E8)

Dá tristeza de saber que um paciente não teve êxito, vai ter que voltar a fazer o tratamento, ou que vai ter que fazer o tratamento só paliativo. (E9)

Também emergiu sentimentos contraditórios, como de alegria frente à cura da doença e a sensação de alívio quando vai a óbito, além de tristeza e impotência.

[...] em um dia ele está com dor e aí a gente tenta ajustar a medicação, conversa com médico assistente, tenta mudar posicionamento, melhorar o colchão da cama [...] no outro, dia ele continua com a mesma dor e essa dor vai piorando [...] impotente em relação ao próprio curso da doença. (E1)

Muitas vezes a gente se sente incapaz de fazer alguma coisa para ajudar. (E3)

Alguns principalmente a gente vê [a morte] como se fosse um alivio para o paciente. Eu tento enxergar por esse lado. (E5)

\section{DISCUSSÃO}

Sabe-se que a assistência em enfermagem não envolve apenas procedimentos e conhecimentos técnicos, mas exige presença, flexibilidade, responsabilidade, além da compreensão solidária. Percebe-se que a interação do enfermeiro com o paciente e os demais envolvidos pode abranger relações de amizade, conversas, abraços e outras formas de aproximação ${ }^{(10)}$. Expressões presentes na fala dos entrevistados mencionam que o cuidar não deve restringir-se às técnicas, mas em uma visão ampliada, incorporar a filosofia do cuidado humanizado que contribui no enfrentamento da doença ${ }^{(11)}$.

Nota-se como ponto marcante nos depoimentos o reconhecimento, pelo enfermeiro, da sua importância não apenas em realizar procedimentos, mas em estar-com o paciente, através do diálogo e da presença.
Diante da humanização destaca-se o movimento de se colocar no lugar do outro, em que o profissional toma consciência e reflete acerca de como gostaria de ser cuidado ${ }^{(12)}$, o que é observado nas falas dos entrevistados quando declaram que isto propicia maior aproximação com o paciente e familiares, apesar de lhes trazer abalo emocional.

A compreensão do câncer pela sociedade, em sua maioria, ainda é de uma doença relacionada à dor, sofrimento e morte. Nesse sentido, cabe ao profissional identificar suas próprias concepções acerca da patologia. Faz-se necessário a reflexão em torno das diversas possibilidades de enfrentamento diante de situações do cotidiano, tendo em vista o dever de oferecer um cuidado seguro e eficaz, capaz de auxiliar o paciente e seus familiares na minimização de seus sofrimentos ${ }^{(13)}$.

Com relação à oncologia infantil esse processo é ainda mais dolorido, pois para o enfermeiro a morte envolve paradigmas e preconceitos culturais negativos, emergindo sentimentos de fracasso e impotência, sendo considerado algo inaceitável por se tratar de criança ${ }^{(14)}$.

A equipe de enfermagem que assiste o paciente oncológico convive com sentimentos diversos, desde o sofrimento até a satisfação profissional. O retorno para um novo tratamento, a impossibilidade da cura, o sofrimento dos pacientes e familiares desestruturam os enfermeiros emocionalmente. Entretanto, ao se deparar com situações em que se obteve êxito no tratamento e alcance da cura os profissionais sentem-se realizados e felizes. É possível apreender, de maneira implícita nos relatos, que a morte é percebida como um sinal de fracasso e a vida como um sinal de glória. Mesmo constituindo-se um fenômeno natural, a finitude reflete o temor pelo desconhecido, porque é vivido enquanto experiência do outro ${ }^{(15)}$.

A dificuldade de lidar com essa questão tem contribuído para gerar situações de estresse, impotência, frustração e revolta. O paciente muitas vezes procura o enfermeiro para

falar de suas questões mais íntimas, levando a situações constrangedoras pelo fato de não terem respostas a todas as questões e pela eclosão de sentimentos intensos ${ }^{(16: 424)}$.

O câncer é relacionado a aspectos negativos, como a dor, o sofrimento, a mutilação; a morte desperta angústia e tristeza em todos os envolvidos no processo $^{(17)}$. Nesse contexto, atenta-se para as contradições apontadas pelos enfermeiros depoentes. Ao assis- 
tirem um indivíduo portador de alguma neoplasia, vivenciam sentimentos de alegria e satisfação diante da cura da doença; a sensação de alívio quando o paciente vai a óbito libertando-se do sofrimento; além de sentimentos de tristeza e impotência diante da aflição e padecimento do outro.

Ao cuidar de pacientes terminais o profissional aproxima de sua própria morte, suas limitações e impotência, o que gera sentimentos de culpa, depressão, ansiedade, tristeza e medo pela própria identificação com o paciente ${ }^{(11)}$. Contudo, nota-se que os profissionais de enfermagem sentem-se valorizados mediante o reconhecimento do seu trabalho, que é expresso pelo ser cuidado e familiar por gestos ou palavras, favorecendo a aceitação das situações por eles vivenciadas ${ }^{(11)}$. Salienta-se que estes se surpreendem ao serem reconhecidos por desempenharem atividades básicas de cuidado, como se apenas procedimentos complexos o valorizassem como um bom profissional. Ressalta-se, no entanto, que o cuidado é mais que um ato, uma vez que representa um momento de atuação com zelo e uma atitude de preocupação, responsabilização, envolvimento afetivo e empatia ${ }^{(18)}$.

Assim, os enfermeiros que lidam com pacientes oncológicos encontram-se amplamente vulneráveis, pois no câncer a extensão real dos acontecimentos relacionados à ideia de morte é mais valorizada e acrescida do simbolismo que a doença carrega ${ }^{(19)}$. Portanto, é necessário ao profissional que atua na especialidade oncológica maior controle mental perante as situações que vivencia rotineiramente, tais como cuidar de pacientes em estado terminal ou que demandam cuidados prolongados, o que resulta em maior aproximação, tanto com o paciente, quanto com seus familiares ${ }^{(20)}$.

Neste contexto uma palavra de conforto, o consolo de uma companhia discreta suficientemente acolhedora na presença com o outro ${ }^{(21)}$ pode qualificar o relacionamento interpessoal entre os enfermeiros, pacientes e familiares que vivenciam o processo de doença neoplásica.

\section{CONSIDERAÇÕES FINAIS}

Ao conhecer as percepções e sentimentos de enfermeiros de um hospital oncológico foi possível perceber como é difícil não se abalar psicológica e emocionalmente frente às exigências que perpassam os cuidados técnico-assistenciais. Constatamos os vínculos com todos os pacientes, visto que com a humanização o profissional precisa estabelecer algum grau de envolvimento para com o ser que demanda seus cuidados e para que essa assistência se dê com qualidade e da forma integral.

Destaca-se a necessidade de maior atenção ao tema Oncologia, abordagem do cuidado ao paciente com câncer em todas as suas dimensões, para que esse trabalhador encontre-se mais preparado para lidar com as demandas as quais irá se deparar na sua trajetória ocupacional. Para isso, é importante que o ensino de Enfermagem sensibilize os estudantes, profissionais e docentes para uma prática de cuidado considerando processo de morte e o morrer natural ${ }^{(22)}$. A construção de redes de relações entre as instituições de ensino e os serviços possibilita aos profissionais já inseridos no mercado de trabalho se atualizar.

Enfim, acredita-se que esta pesquisa pode propiciar reflexão acerca do cotidiano da assistência em saúde e que o benefício maior será direcionado não só aos profissionais com pacientes oncológicos, mas também àqueles que assistem pacientes críticos e que experimentam o cotidiano do cuidado a esse paciente com múltiplas demandas.

\section{REFERÊNCIAS}

1. Ministério da Saúde (BR). Instituto Nacional do Câncer. Ações de Enfermagem para o Controle do Câncer: uma proposta de integração ensino-serviço. Rio de Janeiro; 2008.

2. Ministério da Saúde(BR). Instituto Nacional do Câncer. Estimativa 2012 - Incidência de câncer no Brasil. Rio de Janeiro; 2011.

3. Waldow VR, Lopes MJM, Meyer DE. Maneiras de cuidar, maneiras de ensinar: a enfermagem entre a escola e a prática profissional. Porto Alegre: Artes Médicas Sul; 1995.

4. Pinto MH, Cruz MF, Cesarino CB, Pereira APS, Ribeiro RCHM, Beccaria LM. O cuidado de enfermagem ao paciente oncológico fora de possibilidade de cura: percepção de um grupo de profissionais. Cogitare enferm. 2011;16(4):647-53.

5. Kluser SR, Terra MG, Noal HC, Lacchine AJB, Padoin SMM. Vivência de uma equipe de enfermagem acerca do cuidado aos pacientes com câncer. Rev. Rene. 2011; 12(1):166-72.

6. Bastos JCF, Mohallem AGC, Farah OGD. Ansiedade e depressão em alunos de enfermagem durante o estágio de oncologia. einstein. 2008;6(1):7-12. 
7. Martins J, Bicudo MAV. A pesquisa qualitativa em psicologia: fundamentos e recursos básicos. São Paulo: Centauro; 2005.

8. Ministério da Saúde (BR). Conselho Nacional de Saúde. Dispõe sobre diretrizes e normas regulamentadoras de pesquisa envolvendo seres humanos. Resolução n. 196, de 10 de outubro de 1996. Brasília; 1996.

9. Carvalho AS. Metodologia da entrevista: uma abordagem fenomenológica. Rio de Janeiro: Agir; 1991.

10. Fontes CAS, Alvim NAT. Human relations in nursing care towards cancer patients submits to antineoplasic chemotherapy. Acta Paul. Enferm. 2008;21(1):77-83.

11. Oliveira MCL, Firmes MPR. Sentimentos dos profissionais de enfermagem em relação ao paciente oncológico. REME: rev. min. enferm. 2012;16(1):91-7.

12. Gonzales RMB, Beck CLC, Denardin ML. Cenários de cuidado: aplicação de teorias de enfermagem. Santa Maria: Palloti; 1999.

13. Stumm ENF, Leite MT, Maschio G. Vivências de uma equipe de enfermagem no cuidado a pacientes com câncer. Cogitare enferm. 2008;13(1):75-82.

14. Avanci BS, Carolindo FM, Góes FGB, Netto NPC. Cuidados paliativos à criança oncológica na situação do viver/morrer: a ótica do cuidar em enfermagem. Esc. Anna Nery 2009;13(4):708-16.

15. Carvalho LS, Oliveira MAS, Portela SC, Silva CA, Oliveira ACP, Camargo CL. A morte e o morrer no cotidiano de estudantes de enfermagem. Rev. enferm. UERJ. 2006;14(4):551-7.

16. Kovác MJ. Sofrimento da equipe de saúde no contexto hospitalar:cuidando do cuidador profissional. Mundo Saúde. 2010;34(4):420-9.

17. Silva LC. O sofrimento psicológico dos profissionais de saúde na atenção ao paciente com câncer. Psicol. Am. Lat. 2009;16(1):0-0.

18. Boff L. Saber cuidar. Rio de Janeiro: Vozes; 2000.

19. Avellar LZ, Iglesias A, Valverde PF apud Silva IC. Sofrimento psíquico em trabalhadores de enfermagem de uma unidade de oncologia. Psicol. estud. 2007;12(3):475-81.

20. Rodrigues AB, Chaves EC. Fatores estressantes e estratégias de coping dos enfermeiros atuantes em oncologia. Rev. Latino-Am. Enfermagem 2008;16(1):24-8.
21. Oliveira EA, Santos MA, Mastropietro AP. Apoio psicológico na terminalidade: ensinamentos para a vida. Psicol. estud. 2010;15(2):235-44.

22. Cantídio FS, Vieira MA, Sena RR. Significado da morte e de morrer para os alunos de enfermagem. Invest. educ. enferm. 2011;29(3):407-18. 